\title{
Sensitivity and uncertainty analysis for the tritium breeding ratio of a DEMO fusion reactor with a helium cooled pebble bed blanket
}

\author{
Elena Nunnenmann ${ }^{\mathrm{a}}$, Ulrich Fischer, and Robert Stieglitz \\ Institute for Neutron Physics and Reactor Technology, Karlsruhe Institute of Technology, Hermann-von-Helmholtz-Platz 1, 76344 \\ Eggenstein-Leopoldshafen, Germany
}

\begin{abstract}
An uncertainty analysis was performed for the tritium breeding ratio (TBR) of a fusion power plant of the European DEMO type using the MCSEN patch to the MCNP Monte Carlo code. The breeding blanket was of the type Helium Cooled Pebble Bed (HCPB), currently under development in the European Power Plant Physics and Technology (PPPT) programme for a fusion power demonstration reactor (DEMO). A suitable 3D model of the DEMO reactor with HCPB blanket modules, as routinely used for blanket design calculations, was employed. The nuclear cross-section data were taken from the JEFF-3.2 data library. For the uncertainty analysis, the isotopes H-1, Li-6, Li-7, Be-9, O-16, Si-28, Si-29, Si-30, Cr-52, Fe-54, Fe-56, Ni-58, W-182, W-183, W-184 and W-186 were considered. The covariance data were taken from JEFF-3.2 where available. Otherwise a combination of FENDL-2.1 for Li-7, EFF-3 for Be-9 and JENDL-3.2 for O-16 were compared with data from TENDL-2014. Another comparison was performed with covariance data from JEFF-3.3T1. The analyses show an overall uncertainty of $\pm 3.2 \%$ for the TBR when using JEFF-3.2 covariance data with the mentioned additions. When using TENDL-2014 covariance data as replacement, the uncertainty increases to $\pm 8.6 \%$. For JEFF-3.3T1 the uncertainty result is $\pm 5.6 \%$. The uncertainty is dominated by O-16, Li-6 and Li-7 cross-sections.
\end{abstract}

\section{Introduction}

The Helium Cooled Pebble Bed (HCPB) blanket is one of several types of breeding blanket currently under development in the European Power Plant Physics and Technology (PPPT) programme for a fusion power demonstration reactor (DEMO). An important function of the blanket of DEMO is the capability to breed sufficient tritium. This is described by the tritium breeding ratio (TBR) which is the ratio of the tritium produced in the blanket divided by the tritium consumed in the fusion reactions in the plasma. To calculate the TBR, neutronics codes such as MCNP [1] are employed. As they rely on nuclear cross-section data with finite accuracy, uncertainty analyses need to be performed to estimate the uncertainty margin of the TBR result.

Sensitivity and uncertainty analyses of the TBR of the HCPB blanket module have already been undertaken for a mock-up of a single breeding blanket [2] and for this breeding blanket integrated into the ITER port for blanket testing [3]. TBR calculations without uncertainty analysis were routinely performed for the HCPB blanket integrated into a DEMO sector model [4].

The objective of this work was to calculate the nuclear data uncertainty of the TBR for the DEMO reactor with a full HCPB blanket, using the track length estimator approach of MCNP patched with the MCSEN code [6]. The Sandwich code [7] is used to create sensitivity profiles,

$\overline{\text { a e-mail: elena.nunnenmann@kit.edu }}$ calculate integrated sensitivities, and use covariance data from JEFF-3.2 and other sources to assess uncertainties.

\section{The DEMO geometry with integrated HCPB blanket}

A slightly revised version of the model used in [4] was made available for the present work. It was created from a generic $11.25^{\circ}$ sector CAD DEMO model without blankets, into which the blanket modules where placed on the CAD platform. This model was converted into MCNP geometry using the McCAD [5] automated conversion tool. Into the converted model, the manually created interior structure of the blanket modules was inserted utilising the repeated structure technique available with MCNP.

The CAD DEMO model can be seen in Fig. 1. In toroidal direction there is one full inboard blanket module on the inboard side and 1.5 outboard blanket modules on the outboard side. Their dimensions in radial $\times$ toroidal $\times$ poloidal are approximately $0.6 \mathrm{~m} \times 1.2 \mathrm{~m} \times 1.7 \mathrm{~m}$ for the inboard modules and approximately $0.9 \mathrm{~m} \times 1.5 \mathrm{~m} \times 2 \mathrm{~m}$ for the outboard modules.

A schematic of the HCPB blanket modules can be seen in Fig. 2. The structure is stiffened with an internal grid of helium cooled steel plates, which accommodates the breeder units. Every breeder unit consists of two breeder beds filled with $\mathrm{Li}_{4} \mathrm{SiO}_{4}$ pebbles with $\mathrm{Li}-6$ enriched to 60 at $\%$ contained between u-shaped cooling plates with internal helium cooling channels. The remaining space is 


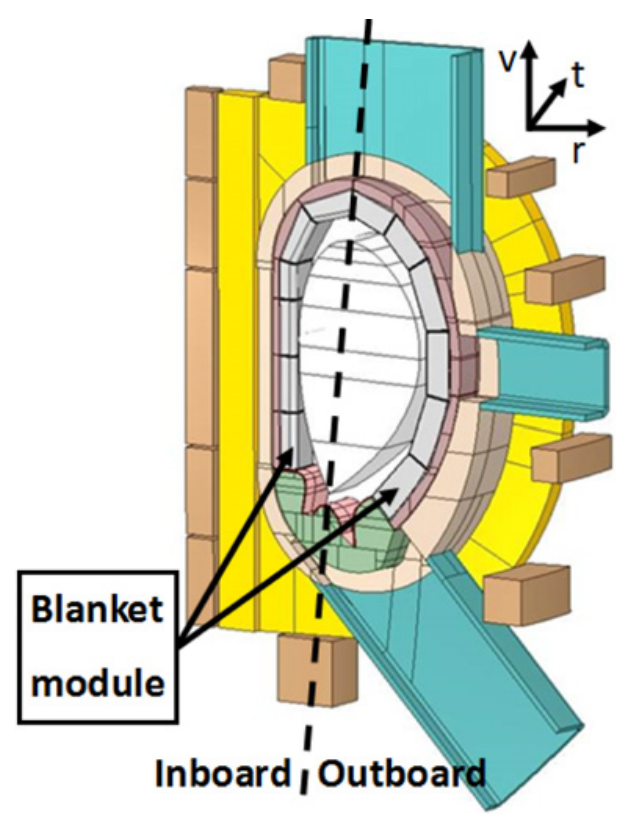

Figure 1. The CAD DEMO $11.25^{\circ}$ sector model.

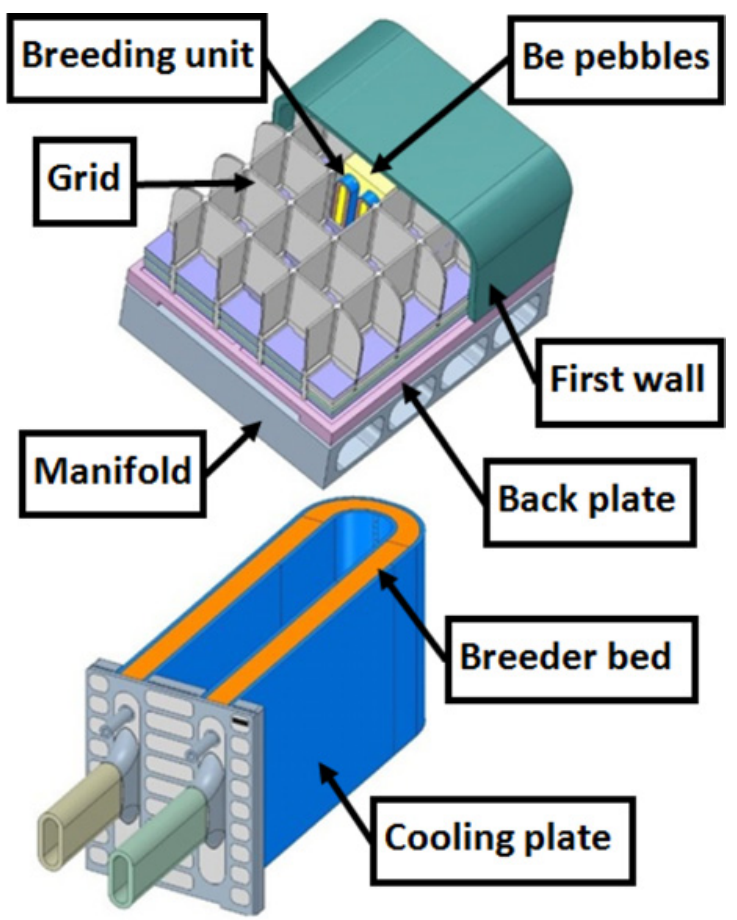

Figure 2. The HCPB blanket module (upper) with the u-shaped breeder unit (lower).

filled with beryllium pebbles for neutron moderation and multiplication.

The resulting neutron spectrum seen by the breeder blankets is displayed in Fig. 3. It has the characteristic peak at $14.1 \mathrm{MeV}$ caused by neutrons coming directly from the plasma. In the range between $10^{-5} \mathrm{MeV}$ till $10 \mathrm{MeV}$ there is a strong, fluctuating neutron flux, caused by neutrons which have undergone interactions with the breeder and other reactor materials.

\section{The sensitivity evaluation}

The TBR was calculated with the track length estimator tally of MCNP with nuclear cross-section data from the

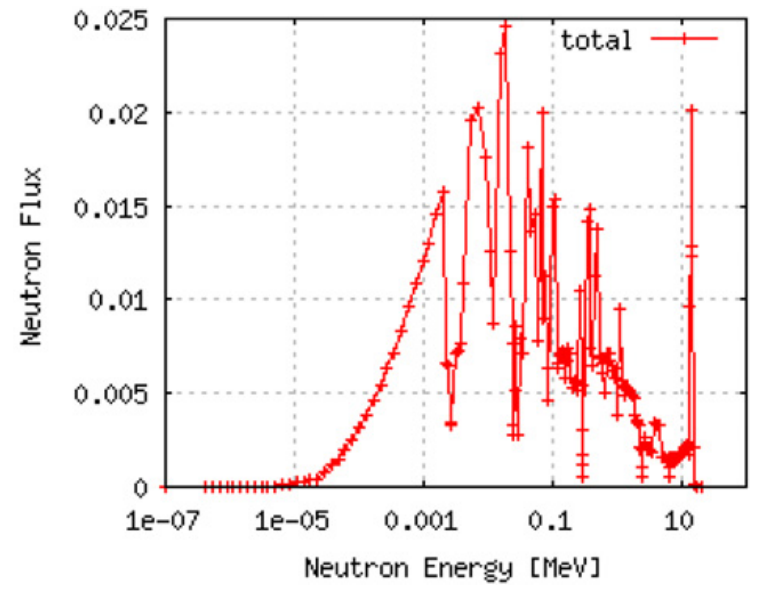

Figure 3. The normalised neutron flux spectrum calculated in the Vitamin-J energy structure averaged over the breeder volume.

JEFF-3.2 library. The sensitivity calculations were performed for $10^{9}$ particle histories with the MCSEN code [6], which is a local update to MCNP and can be installed as a patch. The newest version MCSEN5 was used which is based on MCNP5_1.3. The sensitivities were calculated for every occurence of an isotope in the reactor not only in the breeder materials.

The investigated isotopes were chosen from the breeding material ( $\mathrm{Li}-6, \mathrm{Li}-7)$, the neutron multiplier (Be-9), the breeder ceramic material $(\mathrm{O}-16, \mathrm{Si}-28, \mathrm{Si}-29$, and $\mathrm{Si}-30)$, and the most abundant structural materials (Fe-54, Fe-56, Cr-52, Ni-58, W-182, W-183, W-184, $\mathrm{W}-186$, and $\mathrm{H}-1$ ).

The MCSEN code assumes the TBR does not explicitly depend on the nuclear cross-section, which is true for all cross-sections except the tritium breeding cross-sections of Li-6 and Li-7. In these cases a correction of the MCSEN result needs to be performed with the Add_Response_Term code [7].

Relative sensitivities are calculated by normalising the sensitivity results to the respective Li-6 and Li-7 TBR contribution. Integrating over the whole energy range and adding up the contributions of the different reactions of an isotope yields the total integrated relative sensitivities, which can be seen in Table 1 . The results of $\mathrm{H}-1$ and Ni-58 were not included because they were below $0.001 \% / \%$.

The strongest sensitivity impact comes from O-16, $\mathrm{Li}-6, \mathrm{Si}-28, \mathrm{Li}-7$, and $\mathrm{Be}-9$. It is noted that these are all breeding, breeder ceramic and multiplier constituents, while the impact of structural materials is less strong. Only the less abundant breeder ceramic isotopes $\mathrm{Si}-29$ and $\mathrm{Si}-30$ have a smaller absolute value than the most abundant and highest scoring structural material isotope Fe-56.

For the isotopes with the highest sensitivity results, the strongest individual reactions are displayed as well and it can be seen that for most cases elastic scattering has the strongest impact, followed by inelastic scattering. Only for Be-9 the elastic scattering reaction is in third place after the $(n, 2 n)$ and the $(n, \alpha)$ reaction.

Most sensitivities are negative, which means that an increase of the cross-section would cause a decrease of tritium breeding. Only the sensitivity to the elastic and $(n, 2 n)$ reaction of Be-9 and the tritium breeding reaction of Li-6 is positive. For Li-6 the negative impact of the elastic 
Table 1. The total integrated relative sensitivity of the TBR to the considered isotopes. For the strongest isotopes the results of the strongest individual reactions are displayed as well.

\begin{tabular}{|c|c|c|c|}
\hline isotope & MT & Sensitivity [\%/\%] & Error [\%/\%] \\
\hline \multirow[t]{4}{*}{ Li-6 } & & -0.630 & $5.7 \mathrm{E}-04$ \\
\hline & elastic & -0.600 & $2.8 \mathrm{E}-05$ \\
\hline & $\left(\mathrm{n}, \mathrm{n}^{\prime}\right)$ & -0.035 & $4.5 \mathrm{E}-06$ \\
\hline & $(\mathrm{n}, \mathrm{t})$ & 0.009 & 5.7E-04 \\
\hline \multirow[t]{4}{*}{$\mathrm{Li}-7$} & & -0.435 & $1.9 \mathrm{E}-05$ \\
\hline & elastic & -0.421 & $1.8 \mathrm{E}-05$ \\
\hline & $(n, 2 n)$ & -0.001 & $1.5 \mathrm{E}-07$ \\
\hline & $\left(\mathrm{n}, \mathrm{n}^{\prime}\right)$ & -0.012 & 2.3E-06 \\
\hline \multirow[t]{4}{*}{ Be-9 } & & 0.196 & $4.5 \mathrm{E}-04$ \\
\hline & elastic & 0.017 & 4.4E-04 \\
\hline & $(\mathrm{n}, 2 \mathrm{n})$ & 0.215 & $8.2 \mathrm{E}-05$ \\
\hline & $(\mathrm{n}, \alpha)$ & -0.029 & $3.1 \mathrm{E}-06$ \\
\hline \multirow[t]{4}{*}{ O-16 } & & -2.830 & $1.1 \mathrm{E}-04$ \\
\hline & elastic & -2.777 & $1.1 \mathrm{E}-04$ \\
\hline & $\left(\mathrm{n}, \mathrm{n}^{\prime}\right)$ & -0.028 & 4.3E-06 \\
\hline & $(\mathrm{n}, \alpha)$ & -0.015 & $2.2 \mathrm{E}-06$ \\
\hline \multirow[t]{4}{*}{$\mathrm{Si}-28$} & & -0.497 & $2.2 \mathrm{E}-05$ \\
\hline & elastic & -0.471 & $2.2 \mathrm{E}-05$ \\
\hline & $\left(\mathrm{n}, \mathrm{n}^{\prime}\right)$ & -0.015 & $2.2 \mathrm{E}-06$ \\
\hline & $(\mathrm{n}, \mathrm{p})$ & -0.005 & $8.1 \mathrm{E}-07$ \\
\hline $\mathrm{Si}-29$ & & -0.024 & $1.7 \mathrm{E}-06$ \\
\hline $\mathrm{Si}-30$ & & -0.016 & $9.9 \mathrm{E}-07$ \\
\hline $\mathrm{Fe}-56$ & & -0.105 & $3.4 \mathrm{E}-04$ \\
\hline $\mathrm{Fe}-54$ & & -0.020 & $1.1 \mathrm{E}-04$ \\
\hline $\mathrm{Cr}-52$ & & -0.010 & $8.6 \mathrm{E}-05$ \\
\hline W-182 & & -0.018 & $6.1 \mathrm{E}-05$ \\
\hline W-183 & & -0.020 & 4.4E-05 \\
\hline W-184 & & -0.020 & $6.8 \mathrm{E}-05$ \\
\hline W-186 & & -0.015 & $6.4 \mathrm{E}-05$ \\
\hline
\end{tabular}

and inelastic scattering overlays the small positive tritium breeding result, causing an overall negative sensitivity.

\subsection{Sensitivity profiles}

Sensitivity profiles were calculated in the Vitamin-J energy group structure. The profile of the sensitivity to the elastic scattering reaction of $0-16$, which has the highest integrated sensitivity impact, can be seen in Fig. 4.

There is a negative peak at $14.1 \mathrm{MeV}$, the energy the neutrons have when they come directly from the plasma, both for the Li-6 and the Li-7 contribution to the TBR. The sensitivity of the Li-7 contribution is less strong and only present above the threshold for the tritium producing reaction at $\approx 3 \mathrm{MeV}$. In contrast, the sensitivity of the Li-6 contribution is strongly negative throughout the energy range down to below $10^{-4} \mathrm{MeV}$ where the neutron flux (see Fig. 3) also vanishes.

An increase in the elastic scattering cross-section of O-16 would cause a decrease of tritium breeding both at $14.1 \mathrm{MeV}$ and throughout the lower energy range. Elastic scattering slows down neutrons and the Li-6 tritium breeding cross-section increases towards lower energies. Therefore, the tritium breeding should benefit from elastic scattering. However, when considering Table 1, it can be

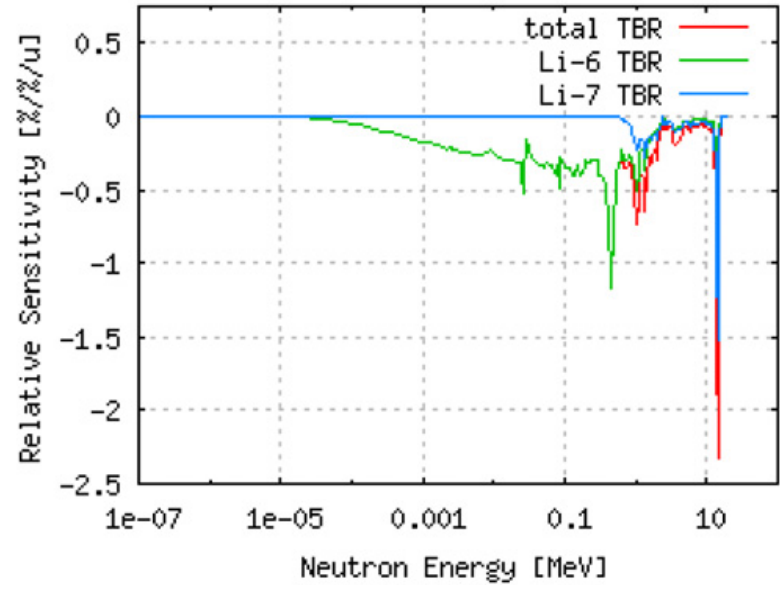

Figure 4. The relative sensitivity profile of the elastic scattering reaction of $\mathrm{O}-16$ for the total $\mathrm{TBR}$, and the $\mathrm{Li}-6$, and $\mathrm{Li}-7$ contribution.

seen that the sensitivity to elastic scattering can both be negative (for $\mathrm{Li}-6, \mathrm{Li}-7, \mathrm{O}-16$ and $\mathrm{Si}-28$ ) and positive (for Be-9). This indicates that it depends on the energy range into which the neutrons are being slowed down. In the case of $\mathrm{Li}-6, \mathrm{Li}-7, \mathrm{O}-16$, and $\mathrm{Si}-28$ it is probable that the neutrons are being slowed down into the energy range of a strong absorbing reaction and for Be-9, this doesn't happen and the tritium breeding can benefit from the slowed down neutrons.

\section{The uncertainty evaluation}

With the sensitivity profiles available from the MCSEN calculations for all the reactions of all the considered isotopes, and, if necessary, the response term correction performed, the Sandwich code [7] can be applied to calculate the resulting relative standard deviations (StDs). To this end, covariance data are required. To achieve consistency with the nuclear cross-section data, the covariance data were taken from JEFF-3.2 for all isotopes for which they are available. For the other isotopes a combination of FENDL-2.1 for Li-7, EFF-3 for Be-9 and JENDL-3.2 for O-16 was used. In addition, covariance data from TENDL-2014 and JEFF-3.3T1 were used for comparison.

The results can be seen in Table 2. H-1 and Ni-58 are not shown as their results were below $0.01 \%$. The total statistical error was $0.0001 \%$ which is significantly smaller than the results for the nuclear data uncertainty.

The different library combinations yield significantly different results: JEFF-3.2 + TENDL-14 yields a result bigger by a factor of almost 3 than JEFF-3.2 + FENDL-2.1 + EFF-3 + JENDL-3.2, and for JEFF-3.3T1 it's a factor of almost 2 . This is caused by the $\mathrm{O}-16$ result being bigger by a factor of 2 for both other libraries and the Li-7 result being bigger by a factor of almost 8 for TENDL-14. The Li-7 result of TENDL-14 is judged as questionable since TENDL data are not very reliable for low mass nuclei. Especially the result for the Li-7 contribution to the TBR has an unrealistically high value of $39.45 \%$.

For all library combinations, $\mathrm{O}-16$ and Li-6 provide the biggest contribution to the total standard deviation (except for the questionable $\mathrm{Li}-7$ value from TENDL-14). Like 
Table 2. Relative StDs of the total TBR calculated with covariance data from JEFF-3.2 + FENDL-2.1 + EFF-3 + JENDL3.2, JEFF-3.2 + TENDL-14 and JEFF-3.3T1.

\begin{tabular}{lccc} 
& $\begin{array}{c}\text { JEFF-3.2 } \\
\text { + other* } \\
\text { rel. StD [\%] }\end{array}$ & $\begin{array}{c}\text { JEFF-3.2 } \\
\text { + TENDL-14* }\end{array}$ & JEFF3.3T1 \\
rel. StD [\%] & rel. StD [\%] \\
\hline Li-6 & 1.25 & 1.25 & 1.25 \\
Li-7 & $0.85^{*}$ & $6.57^{*}$ & 0.85 \\
Be-9 & $0.59^{*}$ & $0.42^{*}$ & 0.59 \\
O-16 & $2.64^{*}$ & $5.29^{*}$ & 5.29 \\
Si-28 & 0.48 & 0.48 & 0.48 \\
Si-29 & 0.11 & 0.11 & 0.11 \\
Si-30 & 0.10 & 0.10 & 0.10 \\
Fe-56 & 0.29 & 0.29 & 0.29 \\
Fe-54 & 0.07 & 0.07 & 0.07 \\
Cr-52 & 0.06 & 0.06 & 0.06 \\
W-182 & 0.08 & 0.08 & 0.08 \\
W-183 & 0.12 & 0.12 & 0.12 \\
W-184 & 0.27 & 0.27 & 0.27 \\
W-186 & 0.14 & 0.14 & 0.14 \\
\hline total & 3.17 & 8.57 & 5.58 \\
\hline
\end{tabular}

Table 3. Absolute standard deviations of the TBR calculated with covariance data from JEFF-3.2 + FENDL-2.1 + EFF-3 + JENDL3.2, JEFF-3.2 + TENDL-14 and JEFF-3.3T1.

\begin{tabular}{lccc}
\hline & Li-6 & Li-7 & total \\
\hline TBR & 1.071 & 0.012 & 1.083 \\
\pm & & & \\
JEFF-3.2+other & 0.034 & 0.0003 & 0.034 \\
JEFF-3.2+TENDL-14 & 0.093 & 0.005 & 0.093 \\
JEFF-3.3T1 & 0.060 & 0.0003 & 0.060 \\
\hline
\end{tabular}

for the sensitivities, structural materials have less impact on the uncertainty than breeder materials. Only the least abundant breeder ceramic isotopes $\mathrm{Si}-29$ and $\mathrm{Si}-30$ yield smaller results than some structural materials.

The result for the TBR was calculated with the track length estimator tally of MCNP. Multiplicating the TBR results with the relative standard deviations yields the absolute standard deviations displayed in Table 3. It can be seen that the standard deviation of the Li-7 contribution does not have an effect on the total standard deviation.

TBR results were also calculated with JEFF-3.2 + TENDL-14 and JEFF-3.3T2 (updated version of JEFF3.3T1). This yielded TBR results of 1.065 and 1.084 respectively, which are well within the range of all of the estimated absolute standard deviations.

\section{Conclusions}

The MCSEN Monte Carlo sensitivity code was successfully applied to the TBR uncertainty calculation of a DEMO reactor model. An $11.25^{\circ}$ torus sector model with a full, integrated HCPB breeding blanket was used.

With the codes Add_Response_Term and Sandwich, the sensitivity data were further processed and combined with covariance data to yield standard deviation results. These results were found to strongly vary depending on the libraries used for the covariance data. The cross-section induced uncertainties vary between $3.2 \%$ for covariances by JEFF-3.2, FENDL-2.1, EFF-3 and JENDL-3.2, 5.6\% for JEFF-3.3T1 and 8.6\% for JEFF-3.2 and TENDL-14.

The breeder ceramic material O-16 and particularly its elastic scattering reaction were found to have the highest impact on the uncertainty of the TBR. Next in line was the main tritium breeding material constituent Li-6 and also the elastic scattering reaction. The sensitivity of the Li-7 contribution to the TBR was found to have no impact on the final standard deviation of the total TBR. Structural materials were found to have less uncertainty impact than breeder materials.

This work was supported by Fusion for Energy (F4E), Barcelona, through the Specific Grant Agreement F4E-GRT-168.02 on "Nuclear Data Improvements and Development of Tools Nuclear Data". The views and opinions expressed herein reflect only the authors' views.

The authors would like to acknowledge the support of Oscar Cabellos for processing covariance data and Reuven Perel for handling the MCSEN, Add_Response_Term and Sandwich code.

\section{References}

[1] Brown, Forrest B and others, Los Alamos National Lab Report: LA-UR-03-1987 (2003)

[2] D. Leichtle, U. Fischer, I. Kodeli, R.L. Perel, M. Angelone, P. Batistoni, P. Carconi, M. Pillon, I. Schäfer, K. Seidel, R. Villari, and G. Zappa, Fusion Engineering and Design 82(15), 2406-2412 (2007)

[3] U. Fischer, D. Leichtle, and R.L. Perel, Fusion Engineering and Design 83(7), 1222-1226 (2008)

[4] P. Pereslavstev, P. Lu, and U. Fischer, Report for TA WP13-SYS-022 (2014)

[5] L. Lu, U. Fischer, and P. Pereslavtsev, Fusion Engineering and Design 89(9), 1885-1888 (2014)

[6] R.L. Perel, J.J. Wagschal, and Y. Yeivin, Nuclear Science and Engineering 124(1), 197-209 (1996)

[7] R. Perel, Task 3.3 of the F4E Grant F4E-FPA-168.01 (2014) 